Revista de Psicología Vol. 36 (1), 2018 (ISSN 0254-9247)

\title{
Factores predictores del bienestar subjetivo en adultos mayores
}

\author{
William Ignacio Torres Palma y Mirta Margarita Flores Galaz ${ }^{2}$ \\ Universidad Autónoma de Yucatán, México
}

El presente estudio evaluó el efecto de las variables satisfacción con la vida, autopercepción de salud, apoyo social y estilos de enfrentamiento sobre el bienestar subjetivo. Se contó con una muestra de 122 adultos mayores mexicanos del Estado de Yucatán, en la ciudad de Mérida con un rango de edad de 60 a 93 años. Los resultados en mujeres muestran que los estilos de enfrentamiento son predictores del bienestar subjetivo en los afectos negativos. En cuanto a la dimensión cognitiva del bienestar subjetivo, la autopercepción de salud, los estilos de enfrentamiento y la satisfacción con la vida fueron predictores adecuados. En hombres, los resultados demuestran que la dimensión cognitiva del bienestar subjetivo fue predicha a partir de la satisfacción con la vida y los estilos de enfrentamiento. Los hallazgos muestran la importancia del profundizar y promover variables psicosociales en los adultos mayores.

Palabras clave: bienestar subjetivo, percepción de salud, estilos de enfrentamiento, satisfacción con la vida, adultos mayores.

\section{Predictive factors of subject well-being in older people}

This study assessed the effect of variables such as life satisfaction, self-rated health, social support and coping styles on subjective well-being. The level of subjective well-being was studied in a sample of Mexican elderly persons from the state of Yucatan in Merida $(n=122)$ with an age range of 60-93 years. The results in women show that coping styles predicts subjective well-being in negative affects. Moreover, self-perception of health, coping styles, and satisfaction with life are adequate predictors of the cognitive dimension of subjective well-being. Results in men show that satisfaction with life and coping styles predict the cognitive dimension of subjective well-being. Findings point to the importance of exploring psychosocial variables in older adults.

Keywords: well-being, self-rated health, coping styles, life satisfaction, older adults.

1 Estudiante del doctorado en Ciencias Sociales de la Universidad Autónoma de Yucatán. Dirección postal: C 1ª no 310 x 36 y 38 col pensiones. C.P: 97219, Mérida Yucatán, México. Contacto: w_torres_1@hotmail.com

2 Doctora en Psicología Social. Profesora investigadora de tiempo completo en la Facultad de Psicología de la Universidad Autónoma de Yucatán. Miembro del Sistema Nacional de Investigadores de México. Dirección postal: KM 1 Carretera Mérida-Tizimín, Cholul. C.P. 97305. Mérida Yucatán, México. Contacto: fgalaz@uady.mx 


\section{Os fatores preditivos de sujeito bem-estar em pessoas mais velhas}

Este estudo avaliou o efeito de variáveis, satisfação com a vida, autopercepção de saúde, apoio social e estilos de coping no bem-estar subjetivo. o nível de bem-estar subjetivo foi estudado no estado de uma amostra de adultos mexicanos mais antiga do Yucatan em Merida $(n=$ 122) com uma faixa etária de 60-93 anos. Os resultados em mulheres mais antiga mostram que os estilos de enfrentamento são preditores de bem-estar subjetivo em efeito negativo em termos da dimensão cognitiva do bem-estar de saúde subjetiva auto-avaliação, estilos e satisfação com a vida de enfrentamento foram preditores adequados em homens mais antiga resultados mostram que a dimensão cognitiva do bem-estar subjetivo está previsto a partir de satisfação com a vida e estilos de enfrentamento.

Palavras-chave: bem-estar subjetivo, percepção de saúde, estilos de enfrentamento, satisfação com a vida, idosos

\section{Facteurs prédictifs du bien-être subjectif chez les personnes âgées}

Cette étude a évalué l'effet de la satisfaction de la vie, de l'auto-perception de la santé, du soutien social et des styles d'adaptation sur les variables de bien-être subjectif. Nous avons étudié le niveau de bien-être subjectif d'un échantillon de 122 Mexicains âgés de l'État du Yucatan, dans la ville de Mérida, âgés de 60 à 93 ans. Les résultats chez les femmes montrent que les styles d'adaptation sont des facteurs prédictifs du bien-être subjectif des affects négatifs, en termes de la dimension cognitive du bien-être subjectif, l'auto-perception de la santé, les styles de confrontation et de satisfaction avec la vie étaient des prédicteurs adéquats, tandis que chez les hommes, les résultats montrent que la dimension cognitive du bien-être subjectif est prévu de satisfaction de la vie et les styles d'adaptation. Les résultats montrent l'importance d'approfondir et de promouvoir les variables psychosociales chez les personnes âgées.

Mots-clés: bien-être subjectif, perception de la santé, les styles de confrontation, satisfaction avec la vie, personnes âgée. 
La vejez es una etapa natural e inherente a todo ser vivo, que es considerada una evolución en el ciclo de vida del ser humano, con cambios de diversa índole, como lo son los psicológicos, biológicos y sociales (Laforest, 1991). Esta etapa de transiciones es influida por la cultura y por la cosmovisión particular de cada persona (Kehl \& Fernández, 2001; Acevedo Aleman \& Gonzalez Tovar, 2014). En ocasiones se considera que la persona vive en el pasado, evocando los recuerdos hacia su presente y futura, perdiendo interés en la prospectiva de la vida (Álvarado García \& Salazar Maya, 2014; Quintero, Henao, Villamil \& León, 2015).

Desde la óptica biológica, el envejecimiento supone un decaimiento de las funciones propias del organismo (Gonzales de Gago, 2010), declive que varía según el estilo de vida llevado con anterioridad. Es decir, el envejecimiento poblacional supone un aumento de patologías y discapacidades (Aparicio, 2003), que ameritan cuidados (Deluigi, 2016). La disminución de la capacidad funcional durante el envejecimiento siempre ha sido una preocupación para la humanidad (Laforest, 1991), sin embargo, esto ha cobrado relevancia desde hace varias décadas, debido a que el mundo está inmerso en una gran metamorfosis poblacional (Roig Hechavarría, Ávila Fernández, Macdonald Bron \& Ávila Fernández, 2015) es decir, la población mundial envejece aceleradamente (Organización Mundial de la Salud, 2015; Rojpaisarnkit, 2016).

El envejecimiento de la población se vincula estrechamente con el desarrollo económico. Las regiones actualmente desarrolladas, como Europa Occidental, Japón, Estados Unidos y Canadá fueron las primeras en pasar por esta transición demográfica (Organización Mundial de la Salud, 2015). El número de habitantes mayores de 60 años se acerca a los 580 millones de personas, de las cuales 380 viven en países desarrollados, en donde la seguridad social solo cubre a la mitad de las personas (Suárez \& Pescetto, 2005). Entre 2000 y 2050, la proporción 
de los habitantes del planeta mayores de 60 años se duplicará, pasando del $11 \%$ al $22 \%$. En números absolutos, este grupo de edad pasará de 605 millones a 2000 millones en el transcurso de medio siglo (Organización Mundial de la Salud, 2015).

En la actualidad los países menos desarrollados son los que experimentan el cambio poblacional, pero de forma acelerada. El nivel de envejecimiento de América Latina emula en 50 años lo que a Europa le tomo dos siglos, con la desventaja de poseer menor tiempo para realizar ajustes para enfrentar el nuevo escenario poblacional (Aranibar, 2001). México como país latinoamericano también está inmerso en esta dinámica poblacional, ya que como indica Wong (2013), México logrará en tan solo 26 años contar con una tasa de población adulta mayor del $15 \%$, cuando en otros países este ritmo ha sido más lento. $\mathrm{Al}$ respecto, en México se contabilizaron 10,222,624 personas mayores de 60 años en el año 2010, lo que significa que los habitantes de la tercera edad representan una proporción de $9.1 \%$ de la población total, misma que, se estima, alcanzará 17 millones en el año 2030 (Instituto Nacional de Estadística, Geografía e Información, 2013).

Un indicador claro del envejecimiento poblacional en México es la transformación de la estructura por edades de la población, en donde la edad media de la población, que pasó de 21.8 años en 1970 a casi 27 años en 2000, es uno de los indicadores que confirma que nuestra sociedad ha entrado en este proceso (Instituto Nacional de Estadística, Geografía e Información, 2013). Se prevé que para 2030 México tendrá una edad media de 37 ańos y de poco menos de 43 en 2050. Por su parte, la esperanza de vida seguirá en aumento, al pasar de 75.2 ańos en 2004 a 76.6 en 2010, 78.5 años en 2020 y 79.8 en 2030 (Secretaría de Gobernación, 2008). En 2000 había en México 20.5 adultos mayores por cada cien personas de cero a catorce ańos; cuatro ańos más tarde esta proporción ha aumentado a 24.4. Se prevé que estas cifras aumenten a 55.1 y 85.7 en 2020 y 2030, respectivamente. Se estima que a partir del año 2034 la proporción se invertirá, de manera que en la población habrá más personas de la tercera edad que menores de quince años (Secretaría de Gobernación, 2008). 
En el caso particular de Yucatán, los datos del Instituto Nacional de Estadística, Geografía e Información (2013, p. 1), muestran que en el periodo comprendido entre 1990 y 2010 la población en su conjunto se incrementó $1.80 \%$ promedio anual, mientras que la del grupo de edad de 60 años o más lo hizo a un 3.07 por ciento. Es así que una de las consecuencias en México de este rápido envejecimiento demográfico poblacional es la incapacidad del sistema para poder atender y asegurar un bienestar a los adultos mayores, por lo tanto, este sector poblacional es caracterizado por la discriminación y vulnerabilidad. Esta discriminación permea todos los ámbitos de la vida que van desde el familiar, social, económico y de participación social (Consejo Nacional para Prevenir la Discriminación, 2010, p. 35).

En este sentido, Garay y Montes de Oca (2011) hacen hincapié en que el escaso desarrollo de México está ligado a la pobreza de los adultos mayores que se ven obligados a seguir en el mercado laboral en condiciones de alto riesgo (Torres Góngora, 2013). Esto reafirma lo señalado por Aranibar (2004) quien afirma que la vulnerabilidad de los adultos mayores no se traduce solamente en términos patológicos, también es claramente definida en el ámbito social. Es así que el adulto mayor debe enfrentar las manifestaciones discriminatorias (Villagómez Váldez, 2014) que son expresadas a través de manifestaciones culturales de marginación y exclusión que en gran medida no han sido combatidas a nivel nacional y estatal (Arroyo Rueda, 2011, Montes de Oca, 2013).

Siguiendo con lo anterior, las condiciones de seguridad social pueden considerarse escasas, ya que en el conjunto de todas las instituciones que componen la seguridad social, esta solo protege a la tercera parte de la población económicamente activa, aquella principalmente asalariada en el sector formal urbano (Ham Chande, 2011). Cabe destacar que México como Estado ha abordado esta situación, desde un enfoque de pobreza e integración social, para el cuál, la respuesta ha girado en torno a brindar servicios de asistencia social (Razo González, 2014).

Para muchos adultos mayores, el panorama no es muy alentador. Sin embargo, hay quienes logran llegar a la vejez de una forma exitosa. Cabe recalcar que, se ha señalado al bienestar subjetivo como 
un elemento fundamental para lograr un envejecimiento positivo y que, desde la perspectiva cuantitativa, es posible conocer y sistematizar aquellas variables o factores que ayudan o promueven condiciones para alcanzar el bienestar subjetivo adecuado (Diener, Kesebir \& Lucas, 2008). Lo anterior, ha despertado el interés a nivel mundial para conocer las condiciones de vida y las necesidades específicas del adulto mayor, cobrando relevancia en los últimos años las investigaciones relacionadas con el bienestar subjetivo del adulto mayor y aquellas variables que influyen como la satisfacción con la vida (Temkin \& Del Tronco, 2006), apoyo social (Vivaldi \& Barra, 2011), autopercepción de la salud y estilos de enfrentamiento.

El estudio del bienestar subjetivo surge ante la necesidad de explorar la forma de potencializar las cualidades y virtudes, que puedan incidir de forma positiva en la calidad de vida de las personas (Csikszentmihalyi, 1998; Diener, 1994; Diener \& Chang, 2011; Seligman, 2013; Steptoe, Deaton \& Stone, 2015) e incorporar estas potencialidades hacia un desarrollo conjunto como Estados y naciones más allá de índices económicos (Chacón, 2015; Salas \& Garzon, 2013), es así, que el bienestar subjetivo incluye el estado emocional en un determinado momento, la satisfacción respecto a cada dominio de la vida, que se desprende de una valoración global respecto a la propia vida (Diener \& Lucas, 1999).

A raíz de esto, múltiples estudios se han dado a la tarea de diferenciar y establecer los componentes que conforman el bienestar subjetivo y en qué medida corresponden a la realidad empírica (Triado, 2003), como resultado de lo anterior se ha llegado a cierto consenso, el cual manifiesta que existen dos dimensiones del bienestar subjetivo, un componente afectivo que incide en el estado emocional de la persona en lapsos cortos de tiempo (Diener, 2000) y un componente cognitivo que se refiere a la valoración que realiza la persona sobre los diversos dominios de su vida a lo largo de su trayectoria de vida, al ser una evaluación de toda la vida, los adultos mayores pueden concretar esta evaluación de formas más complejas que las personas más jóvenes (Blanch, Sahagún, Cantera \& Cervantes, 2010; Diener \& Lucas, 1999;Diener, Lucas \& Suh, 1996). 
Dentro de esta línea de investigación, una gran parte de científicos sociales (Ramiro, Navarro, Menacho Jiménez, Mar López \& GarcíaSedeńo, 2016) se han ocupado de estudiar los aspectos que llevan a una persona a evaluar positivamente su existencia. Es así que Diener (1994) señala tres elementos del bienestar: subjetividad, presencia de indicadores positivos/ausencia de factores negativos y valoración global de la vida. De esta manera un alto bienestar depende de una apreciación positiva de la vida que perdura a lo largo del tiempo, mientras que el bajo nivel obedece el desajuste entre expectativas y logros que correspondería a un elemento cognitivo. El componente afectivo constituye el plano hedónico, es decir, el que contiene el agrado experimentad (Carrasco-Campos, Martínez Fernández \& Moreno Mínguez, 2013) por la persona con sus sentimientos, emociones y estados de ánimo más frecuentes. En este punto cabe resaltar que en el término bienestar subjetivo, se considera la dimensión empíricamente medible de la felicidad, y difiere del concepto de calidad de vida, al incluir autoevaluaciones y no solo estándares explícitos, que son operacionalizados a través de componentes objetivos (Veenhoven, 2012).

Entre las variables estudiadas con el bienestar subjetivo se encuentra el nivel de ingreso y la pobreza. Diener y Biswas (2002) señalan que la relación entre ingreso y bienestar subjetivo solo es perceptible si la persona se encuentra en una situación de extrema pobreza, resaltando que las variables psicosociales son consideradas el origen de las diferencias en el bienestar subjetivo y cobran relevancia cuando las necesidades básicas son cubiertas, lo que evidencia la incapacidad de explicar en su totalidad el bienestar subjetivo a partir de variables exclusivamente económicas.

Las investigaciones del bienestar subjetivo en adultos mayores han realizado diversos correlatos con múltiples variables. Los estudios han revelado asociaciones con el locus de control, en donde un locus de control interno puede contribuir a una disposición interna para adaptarse a las circunstancias de la vida (Palomar Lever \& Valdéz Trejo, 2004). De igual forma variables como la autoestima (Olvera, 2015) y la autoeficacia parecen afectar el bienestar subjetivo, Diener y Diener 
(1995) explican que la autoestima decae al experimentar eventos desafortunados lo que influye negativamente en el bienestar subjetivo, sin embargo, estos efectos pueden ser incrementados por una cultura individualista en donde los sentimientos de valía de uno mismo son más importantes que en una cultura donde se valore lo colectivo

En cuanto a los estudios del bienestar subjetivo y la edad, indican que la edad es una variable a tomar en cuenta ya que a mayor edad, mayor bienestar subjetivo (Kunzman, Little \& Smith, 2000), esta asociación es mayor, cuando las variables ingreso y salud son controladas, de tal forma esta relación se enfoca en la evaluación cognitiva y se relaciona negativamente con la afectividad negativa y positiva (Herzog \& Rodgers, 1981), en donde existe un ligero decremento (Diener \& Lucas, 2000), sin que signifique un deterioro del bienestar subjetivo (Pinquart \& Sörensen, 2000), debido a que el componente cognitivo tiene un efecto a largo plazo en el bienestar subjetivo, a diferencia de los afectos (Diener \& Suh, 1998).

En un estudio en México, Vera Noriega, Sotelo Quiñones y Domínguez Guedea (2005) confirman los altos índices de bienestar subjetivo en los adultos mayores, estos datos hablan de la estabilidad en cuanto a la evaluación de la vida por parte de los adultos mayores, la cual explica García Martín (2002), quien señala que la estabilidad de la satisfacción vital a lo largo de los años se debe a una disposición natural en las personas a adaptarse a sus circunstancias.

Ante esto, el enfrentamiento es un mecanismo que permite a la persona adaptarse ante los eventos estresantes o amenazantes en su entorno, de ahí la importancia de esta variable para la predicción del bienestar subjetivo, asimismo existen otras variables predictoras como el apoyo social (Anguas, 1997; García Martín, 2002; Tu \& Yang, 2015) y la salud percibida, que resultan fundamentales en el bienestar subjetivo (Aranibar, 2001; Mella, Apolonio, Maldonado, Fuenzalida \& Díaz, 2004).

Los estilos de enfrentamiento se refieren a predisposiciones personales para hacer frente a las situaciones y son los responsables de las preferencias individuales en el uso de unos u otros tipos de estrategia 
de enfrentamiento, así como de su estabilidad temporal y situacional (Pelechano, 2000). En este sentido, se señala que envejecer en forma exitosa o competente depende fundamentalmente de las respuestas adaptativas que es capaz de dar el adulto mayor, ante los cambios físicos que van ocurriendo con el paso del tiempo y frente a los factores psicosociales estresantes que se van presentando en esta etapa del ciclo vital (Fernández-Ballesteros, Moya Fresneda, Iñiguez Martínez \& Zamarrón Cassinello, 1999; Zúñiga \& García, 2008; González-Celis \& Araujo Viveros, 2010). Cabe mencionar que no todos los estilos y estrategias de enfrentamiento producen beneficios, es decir pueden ser contraproducentes y ser una amplificación del estrés (Davis \& Palladino 2008). Un punto a considerar en relación con el enfrentamiento es que los adultos mayores tienden a enfrentarse a los problemas de forma algo distinta que los adultos jóvenes (Stefani, 2004). En lo general, los adultos mayores se enfrentan a los problemas utilizando soluciones centradas en la emoción más que en el problema mismo. Las respuestas de tipo emocional son más pasivas que confrontativas, más personales y más orientadas al control de los sentimientos negativos que a la modificación de las circunstancias del problema.

En relación con lo anterior, Folkman, Lazarus, Pimley y Novacek (1987) explican que los adultos mayores tienen mayor capacidad para tomar distancia con respecto a las situaciones estresantes y revaluarlas positivamente, lo que permite centrarse en la emoción. De igual forma, los mecanismos de enfrentamiento que tomen los adultos mayores para ser frente a los problemas o a una situación amenazante para su vida, van a depender del contexto donde habitan el cual pueden ser un asilo o residencia geriátrica y su domicilio (Stefani \& Feldberg, 2006). En este sentido, existen estudios que señalan que aquellos adultos mayores que residen en sus hogares poseen recursos que les permiten adaptarse con mayor eficiencia, características como la vida independiente y el hogar propio proporcionan una percepción de control que permite desplegar estrategias de enfrentamiento adaptativas haciendo frente al problema (Stefani \& Feldberg, 2006). 
Reyes Rasse y Rojas Arellano (2006) señalan que es necesario analizar los estilos y estrategias de enfrentamiento en adultos mayores, ya que se perciben grandes diferencias entre los adultos institucionalizados y los no institucionalizados, en donde los adultos mayores no institucionalizados utilizan menos estrategias de enfrentamiento, se centran en planificar, en la búsqueda de apoyo social, además realizan un encuadre positivo a las situaciones; por su parte, los adultos mayores institucionalizados afrontan las demandas de la vida a través de un enfrentamiento activo, aceptación de la responsabilidad y autocontrol.

Otros estudios señalan que los adultos mayores, en comparación con los adultos jóvenes, están más frecuentemente sometidos a situaciones problemáticas que están fuera de su control, como la enfermedad crónica, la muerte de familiares y amigos, la discapacidad y la cercanía de la propia muerte (Solomon, 1996), y utilizan en forma predominante las estrategias que se centran en el control del distrés emocional asociado a la situación (González-Celis \& Padilla, 2006). Esto último, es debido a que los ancianos evalúan a los acontecimientos estresantes como inmodificables en mayor proporción que los adultos (Castro Bolaño et al., 1995).

De esta forma, el enfrentamiento representa un eje fundamental para el adulto mayor, ya que rige la forma de tratar aquellas situaciones que pueden resultar estresantes o causantes de malestar que de alguna forma pueden incidir positiva o negativamente en el bienestar subjetivo, es decir en la evaluación de la vida. El enfrentamiento y el bienestar subjetivo poseen un origen a partir de la percepción individual de la persona, en el caso del enfrentamiento depende principalmente de la percepción de las amenazas del ambiente (Lazarus \& Folkman, 1984) y el bienestar subjetivo se basa en una valoración cognitiva y afectiva de la vida (Diener, 1994). En este sentido, Folkman y Moskowitz (2004) refieren sobre la efectividad de las estrategias de enfrentamiento: el adecuado ajuste entre las demandas de la situación y la forma de enfrentarla, junto a la capacidad de la persona para modificar su respuesta en función de estas demandas. Así, los enfrentamientos no son en sí mismos efectivos o no, su efectividad estará en función del contexto y del 
modo específico en que se pongan en juego. Es decir, para los adultos mayores, las pérdidas de diversas índoles no afectan directamente el bienestar subjetivo, sino la forma de vivirlo, aquí es donde el enfrentamiento se vuelve un mediador de las exigencias o estresores ambientales y las evaluaciones sobre la vida que realice la persona (Sandin, 2003). En un estudio en México sobre adultos mayores se demostró que un estilo de enfrentamiento en donde se valore lo positivo contribuye de forma positiva al bienestar subjetivo (Vera Noriega, Sotelo Quiñones \& Domínguez Guedea, 2005).

Bajo la premisa anterior, se considera que los estilos de enfrentamiento presentan contribuciones significativas al bienestar subjetivo, ya que son mecanismos que los individuos utilizan para minimizar los efectos del estrés (Figueroa, Contini, Lacunza, Levín \& Estévez, 2005; Cano, Rodríguez \& García, 2007), disminuyendo los afectos negativos y permitiendo una evaluación positiva en términos de vida.

En conclusión, los estilos y estrategias de enfrentamiento pueden tener un impacto positivo en el adulto mayor, incidiendo en muchas esferas de la vida, logrando que tenga un mejor rendimiento social y desarrollando habilidades que le permitan valerse por sí mismo, a su vez esto incidirá positivamente en las evaluaciones que realiza el adulto mayor sobre sus metas y objetivos, lo que se relaciona con el bienestar subjetivo (González-Celis \& Padilla, 2006).

Por su parte, Fernández-Ballesteros (1996), destaca la gran influencia positiva del apoyo social, derivado de una red de interacción, con el bienestar subjetivo; de esta forma los vínculos y la satisfacción con estos, son un factor imprescindible para lograr un envejecimiento exitoso. En este punto se recalca la importancia de esta actividad social, pero no es la actividad por sí misma que incide en el bienestar, sino en la valoración subjetiva que es construida con relación a los otros, en un espacio social determinado (Muñoz Muñoz, 2013).

Al hablar de valoraciones subjetivas surge el concepto de apoyo social percibido, que está formado por las evaluaciones subjetivas de la ayuda dada y/o recibida (Hofer \& Sliwinski, 2006). Las investigaciones sobre el concepto de apoyo social sugieren que sus efectos son 
los que tienen mayor incidencia sobre la salud y el bienestar en la vejez (Krause, 1997). De tal forma que se considera el predictor más relevante del bienestar subjetivo de las personas mayores, sobre todo si lo que se mide es la satisfacción con el apoyo recibido. Lepore, Evans y Schneider (1991) especifican la forma en que el bienestar subjetivo es afectado por el apoyo social percibido (Svensson, Martensson \& Hellstrom, 2012), al señalar que los adultos mayores que perciben que su red les da mucha ayuda, serán más resistentes a los estresores ambientales que aquellos que no se sienten ayudados de la manera esperada. Además, el apoyo percibido puede usarse para detectar la presencia de interacciones sociales negativas, intercambios desiguales e insatisfacción por la falta de apoyo o la inadecuación de la ayuda, así como para predecir futuros problemas de salud (Krause, Liang \& Yatomi, 1989; Newsom, Mahan, Rook \& Krause, 2008).

Con relación al apoyo social percibido, Vera y Sotelo (2004) realizaron un estudio en donde evaluaron las fuentes informales de apoyo (familia y amigos), además del apoyo recibido y dado, concluyendo que las evaluaciones positivas de estos recursos se relacionan estrechamente con el bienestar en los adultos mayores. Por otro lado, la evidencia empírica, ha encontrado que el apoyo social, que proviene de una diversidad de fuentes, en lugar de solo una o dos, incide con mayor profundidad en el bienestar de los adultos mayores (Salinas, Manrique \& TéllezRojo, 2008). Arias (2008) complementa al mencionar que una red de apoyo tan limitada y poco diversificada pueden restringir otros tipos de apoyo, principalmente los instrumentales y económicos. Este hallazgo es importante, ya que como menciona Montes de Oca (2003), la mayoría de los adultos mayores está inmerso en una red familiar, debido al debilitamiento de las redes de vecinos o amigos. Salinas, Manrique y Téllez-Rojo (2008), hacen evidente esta situación en México, ya que sus datos demuestran que las redes sociales de los adultos mayores, está compuesto principalmente por dos vínculos, en su mayoría por familiares que viven en un área cercana o en la misma localidad.

Por otro lado, otra variable de suma importancia para el bienestar subjetivo es la percepción de salud, que se refiere a una valoración 
individual y global que realiza la persona sobre su salud y de las creencias relativas a la salud (medición subjetiva), es un indicador que refleja la condición multifactorial de salud del cual se ha hablado ya, y que engloba el bienestar integral de una persona, sin limitarse solo a las alteraciones fisiológicas o clínicas (Salinero Fort, 2013). Esta valoración personal y subjetiva de la salud, es una característica multidimensional de la persona, dado que cada quien valora su estado de salud en función de una serie de atributos que pueden ser el dolor, el estado emocional, la fatiga, las capacidades visuales o auditivas, la movilidad, la capacidad de relacionarse socialmente, además este juicio tiene implicaciones culturales y sociales (Romero, 2005) y a partir del análisis en conjunto de todos estos factores se realiza la evaluación de la salud. Por ello, la autopercepción de salud es un parámetro que encierra una percepción integral de las personas sobre su salud y constituye un indicador complementario de otras evaluaciones como la función física, el rol físico y el dolor corporal para evaluar en su totalidad la salud física (Hebrero Martínez, 2010).

El concepto de salud de los adultos mayores más que en otros grupos de edad, implica subjetividad (García Vigara, 2015), ya que depende de la conjunción de los efectos de las condiciones fisiológicas, habilidades funcionales, bienestar psicológico y soporte social (Gallegos, García, Reyes \& Durán, 2006; Stein, 2016). No obstante, su determinación más allá de lo estrictamente médico es relevante para los clínicos, los tomadores de decisiones y los investigadores que se interesan en este grupo de la población.

De igual forma la autopercepción de la salud es elemento base en el funcionamiento integral del adulto mayor, en donde se generan transiciones de tipo psicosocial, que afectan el estilo de vida por el incremento de agentes que acompañan esta etapa, como son el contexto, la familia, los factores económicos o el nivel de pobreza (Caicedo \& Berbesi Fernández, 2014), los cambios culturales y políticos y todas aquellas acciones que directa o indirectamente aluden a la salud percibida. Es así que la percepción de salud en el adulto mayor se convierte en un objeto central de investigaciones, ya que provee información que no se limita 
al estado de salud, sino también del gran número de factores que se asocian a ellas. Estudios en mujeres indican que la percepción del estado de salud o salud subjetiva es un indicador del estado de salud global y es un importante predictor de las necesidades asistenciales y líneas de organización de programas (Cerquera Córdoba, Flores Jaimes \& Linares Restrepo 2010). En esta misma línea, Vargas Esquivel (2002) ha encontrado una asociación positiva entre la salud percibida y el nivel de independencia, es decir que a mayor independencia del adulto mayor se genera una valoración positiva de la salud.

Por otra parte, la salud percibida y el bienestar subjetivo se encuentran fuertemente relacionados de forma empírica, es así que estos indicadores en conjunto proveen un panorama más amplio de la condición del adulto mayor (Mendoza Sierra, 1996). La salud percibida es un parámetro fundamental en la forma que el adulto mayor valora sus experiencias cognitivas y afectivas de su vida en general, lo que da lugar al bienestar subjetivo, siendo el principal determinante de la percepción de salud, el nivel de actividad que puede generar el adulto mayor (González Celis, 2005). En un estudio, a principios de la década de 1980, Mancini y Orthner (1990) concluyen que la capacidad de realizar actividades físicas y lúdicas es lo que determina la importancia de la percepción de la salud en el bienestar subjetivo de las personas.

Bajo esta premisa, Gwozdz y Sousa Poza (2009) en un estudio con adultos mayores en Alemania, encontraron que la percepción de salud es un mejor predictor de la satisfacción con la vida y bienestar subjetivo, que los indicadores objetivos de la salud, en concordancia a lo anterior las enfermedades crónicas no fueron mejores predictores de la satisfacción con la vida y el bienestar subjetivo (Aranda \& Horna, 2006). Es así, que el juicio o valoración personal que la persona posee de su salud, en conjunto al nivel de importancia que la persona le concede, tiene gran influencia en el bienestar del adulto mayor, de tal forma que, a medida que la persona se considere sana, será capaz de realizar actividades satisfactorias y que influyan en una valoración positiva de la vida (Florenzano. \& Dussaillant, 2012; Salinero Fort, 2013). 
Ante esto, el propósito del siguiente estudio es analizar la capacidad predictiva de la Satisfacción con la Vida, Estilos de enfrentamiento, el Apoyo social y la Percepción de salud en el Bienestar subjetivo en Adultos mayores de la ciudad de Mérida, Yucatán.

\section{Método}

El presente estudio descriptivo de campo, realizado en el ambiente natural, consideró un diseño de dos muestras independientes, divididas por sexo.

\section{Participantes}

El muestreo fue no probabilística intencional y los participantes fueron 122 adultos mayores habitantes de la Ciudad de Mérida, Yucatán. Un criterio de exclusión fue que el participante presentara situaciones físicas o mentales que le impidiesen contestar los instrumentos. La edad de los participantes fluctuó en un rango de edad de 60 a 93 años con una edad promedio de 71.5 y una desviación estándar de 8.4 años. Del total de la muestra el $54.9 \%$ fueron mujeres y el $45.1 \%$ fueron hombres. Asimismo, el $45.1 \%$ estaba casado, el $40.2 \%$ era viudo(a) y el $14.7 \%$ era soltero(a). La totalidad de los participantes cuentan con una jubilación o pensión que los ubica en la clase socioeconómica media. Para la selección de los participantes se acudió a los principales puntos de reunión de los adultos mayores, como templos o iglesias, institutos recreativos y centros de medicina gerontológica.

\section{Medición}

La variable Bienestar Subjetivo fue medida por medio de la Escala Multidimensional para la Medición del Bienestar Subjetivo llamado EMMBSAR (Anguas Plata \& Reyes Lagunes, 1999, 2000) que fue construida para la población mexicana. Esta escala incluye dos dimensiones: Experiencia emocional y evaluación sociocognitiva. Los resultados obtenidos por los autores en un análisis factorial 
exploratorio mostraron que la experiencia emocional mide los aspectos emocionales y afectivos, y está compuesto por diez factores: afectividad positiva $(\alpha=.85)$, expresividad negativa $(\alpha=.87)$, afectividad negativa $(\alpha=.83)$, esperanza $(\alpha=.83)$, desesperanza $(\alpha=.83)$, afectividad interna positiva $(\alpha=.81)$, hedonismo emocional $(\alpha=.83)$, nerviosismo $(\alpha=.83)$, depresión $(\alpha=.82)$ y éxito $(\alpha=.81)$. En la dimensión de evaluación afectivo-cognitiva se incluyen ocho factores: clima familiar $(\alpha=.89)$, interacción social percibida $(\alpha=.85)$, logros $(\alpha=.84)$, calidad de vida $(\alpha=.76)$, interacción social expresada $(\alpha=.64)$, logros nacionales $(\alpha=.81)$, religión $(\alpha=.80)$ y educación $(\alpha=.77)$. Los índices de confiabilidad interna de este instrumento son: Para la experiencia emocional es de $\alpha=.90$, explicando $72.45 \%$ de la varianza y en la dimensión evaluación afectivo-cognitivo es de $\alpha=.94$, cuyos factores explican el $61.38 \%$ de la varianza. Es importante señalar que dado la longitud del instrumento y el tipo de muestra se consideró pertinente contar con una versión corta del instrumento, de manera que se utilizaron los primeros cinco reactivos con el peso factorial más alto de cada factor.

Asimismo, para este estudio se excluyó el factor logros nacionales y calidad de vida, por no tener relevancia teórica para la población estudiada. De ahí que la escala para el estudio quedó conformada por 47 reactivos para ambas subescalas, en donde la experiencia emocional quedo conformada por 10 dimensiones en un continuo con puntuaciones que van del uno al siete midiendo intensidad y frecuencia emocional, y la subescala correspondiente a la evaluación afectiva-cognitiva quedo conformada por seis dimensiones con puntuaciones que van del uno al siete, ambas subescalas se encuentran en formato tipo Likert pictográfico.

Para explorar la variable Satisfacción con la Vida, se utilizó la Escala de Satisfacción con la Vida (Diener, 1994), la versión traducida al español. La escala mide la satisfacción con la vida de forma global y consta de cinco reactivos que poseen puntuaciones que van del uno al siete. La versión original de la SWLS ha mostrado, en diferentes estudios empíricos, propiedades psicométricas adecuadas. Diener, Emmons, Larson y Griffin (1985) encontraron una consistencia interna de .87, 
extrayendo un único factor mediante un análisis factorial exploratorio de ejes principales que explicaba el 66\% de la varianza total.

En cuanto a la variable Apoyo social se administró la Escala de Apoyo Social Percibido llamada CASPE (Calvo Francés \& Díaz Palarea, junio, 2004). Los autores señalan que el análisis factorial reveló tres factores: amistad $(\alpha=.83)$, ausencia de soledad $(\alpha=.81)$, familia y esfuerzo $(\alpha=.57)$ que explican en conjunto el $62.50 \%$ de la varianza total acumulada. La escala consta de 13 reactivos que, evalúan: la existencia de pareja y calidad de la relación, relaciones familiares en sus aspectos cuantitativos y cualitativos, las relaciones de amistad y compañerismo en sus aspectos cuantitativos y cualitativos, además de la participación en organizaciones socioculturales, esto se representa con valores que van del uno al cuatro. Se observan valores aceptables a nivel de consistencia interna $\alpha=.81$ y para la fiabilidad según el método de dos mitades, exhibiendo un $\alpha=.72$.

La variable Autopercepción de salud fue explorada por la Escala Autopercepción de Salud en el Adulto Mayor (Wong, 2013) que fue diseñada para la población mexicana. La escala cuenta con cinco reactivos en un formato tipo, en donde la persona evalúa su salud en relación a sus propias expectativas y en comparación con otras personas. Este instrumento posee validez de contenido.

Para conocer la variable Estilos de Enfrentamiento se aplicó la Escala Multidimensional y Multisituacional de Estilos de Enfrentamiento (Góngora Coronado \& Reyes Lagunes, 1999, 2000) que fue construida para la población mexicana. Este instrumento mide una dimensión duradera y estable de la vida es decir, el enfrentamiento como rasgo, y seis situaciones problemas del enfrentamiento como estado: salud, pareja, escuela, amigos, familia y trabajo. Para este estudio debido al objetivo y el tipo de muestra únicamente se hizo uso del estilo de enfrentamiento como rasgo, es decir el enfrentamiento a la vida. Esta situación consta de 18 reactivos en un formato tipo Likert pictográfico en un continuo de siete puntos, que miden cuatro factores: estilo directo revalorativo $(\alpha=.82)$, emocional-negativo $(\alpha=.74)$, evasivo $(\alpha=.68)$ y apoyo social $(\alpha=.58)$. Su índice de consistencia interna global es de $\alpha=.74$. 


\section{Procedimiento}

Los instrumentos se aplicaron en la ciudad de Mérida, Yucatán, de forma individual. En un primer punto se les pedía su colaboración al adulto mayor resaltando el objetivo del estudio, para lo cual se obtenía el consentimiento informado, mencionando la confidencialidad de los datos, posteriormente se realizaba la administración de los instrumentos, la cual duraba aproximadamente de una a dos horas, siempre monitoreando el estado físico del participante para no causar una sobrecarga física o emocional. La aplicación se realizó en un periodo que comprende de agosto del 2015 a enero de 2016. El Comité Tutoral del Doctorado en Ciencias Sociales de la Universidad Autónoma de Yucatán fue quien aprobó satisfactoriamente las implicaciones éticas del estudio.

\section{Análisis de datos}

El análisis estadístico se realizó a través del Paquete Estadístico SPSS (Statistical Package for Social Sciences) en su versión 23.0. En primer lugar, se verificó la consistencia interna de las pruebas a través del coeficiente Alfa de Cronbach, asimismo, con el objetivo de describir cada uno de los factores de las escalas utilizadas, se realizó un análisis descriptivo por medio de las medidas de tendencia central (medias) y dispersión (desviación estándar). Finalmente, para conocer los mejores predictores del bienestar subjetivo, se realizó un análisis de regresión múltiple (paso a paso) para hombres y mujeres.

\section{Resultados}

En la tabla 1 se observan los resultados descriptivos de la dimensión de experiencia emocional de la EMMBSAR. En este caso los factores Afectividad Positiva $(M=5.18)$ y Afectividad Interna Positiva $(M=5.04)$ fueron los que obtuvieron las medias más altas, en contraste Emocionalidad Negativa $(M=4.57)$ fue la que presento la media más baja, es importante señalar que dicha media está por arriba de la media teórica 
$(M=4)$. En cuanto a la dimensión Evaluación Afectivo-Cognitivo los factores Interacción Social Expresada $(M=6.84)$ y Atributos y Logros $(M=5.69)$ fueron los que obtuvieron las medias más altas, asimismo es importante mencionar que todos los factores tuvieron una media por arriba de la media teórica $(M=4)$, lo cual muestra una percepción positiva del bienestar subjetivo en estas dimensiones.

\section{Tabla 1}

Medias, desviaciones estándares y consistencias internas obtenidas para las dimensiones del Bienestar Subjetivo

Bienestar subjetivo

Dimensión experiencia emocional Dimensión cognitiva

\begin{tabular}{|c|c|c|c|c|c|c|c|}
\hline Factores & $M$ & $D E$ & $\alpha$ & Factores & $M$ & $D E$ & $\alpha$ \\
\hline $\begin{array}{l}\text { Afectividad } \\
\text { positiva }\end{array}$ & 5.18 & .64 & .79 & Atributos y logros & 5.69 & .51 & .77 \\
\hline $\begin{array}{l}\text { Expresividad } \\
\text { negativa }\end{array}$ & 4.65 & .51 & .58 & Clima familiar & 5.65 & .65 & .78 \\
\hline $\begin{array}{l}\text { Expresividad } \\
\text { emocional interna } \\
\text { negativa }\end{array}$ & 4.58 & .55 & .74 & $\begin{array}{l}\text { Interacción social } \\
\text { expresada }\end{array}$ & 6.84 & .51 & .77 \\
\hline $\begin{array}{l}\text { Emocionalidad } \\
\text { negativa }\end{array}$ & 4.57 & .68 & .52 & Religión & 5.64 & .80 & .72 \\
\hline $\begin{array}{l}\text { Afectividad } \\
\text { interna positiva }\end{array}$ & 5.04 & .57 & .59 & $\begin{array}{l}\text { Interacción social } \\
\text { recibida }\end{array}$ & 5.43 & .71 & .80 \\
\hline Desesperanza & 4.60 & .58 & .61 & Educación & 5.36 & .66 & .61 \\
\hline Nerviosismo & 4.62 & .63 & .59 & & & & \\
\hline $\begin{array}{l}\text { Hedonismo } \\
\text { emocional }\end{array}$ & 4.79 & .46 & .45 & & & & \\
\hline $\begin{array}{l}\text { Afectividad } \\
\text { negativa }\end{array}$ & 4.63 & .59 & .62 & & & & \\
\hline Éxito & 4.84 & .51 & .44 & & & & \\
\hline
\end{tabular}


Como se puede observar en la tabla 2 los adultos mayores demostraron un bajo de nivel de Autopercepción de Salud $(M=2.95)$ que dentro del continuo del 1 al 5, dicho puntaje se encuentra por debajo de la media teórica $(M=3)$. En relación al Apoyo Social, los participantes manifestaron sentirse acompańados la mayor parte del tiempo al ubicar el factor de Ausencia de Soledad ( $M=3.03)$ como el factor con la media más alta, que también dentro del continuo del 1 al 4 se encuentra por arriba de la media teórica $(M=2.5)$, en contraposición a lo anterior los adultos mayores revelaron poseer bajos niveles de Amistad $(M=1.87)$. Es decir, se sienten acompañados, pero poseen escasos vínculos con personas que consideren sus amigos. Por otra parte, los adultos mayores poseen un alto grado de satisfacción con la vida, con una media de 4.93, que dentro del continuo de 1 a 7 muestra que están ligeramente por arriba de la media teórica $(M=4)$. Finalmente, los adultos mayores intentan resolver los problemas resaltando el aspecto positivo y llevándose un aprendizaje de la situación, esto se refleja en el factor Directo-Revalorativo $(M=5.60)$ que fue el que obtuvo la media más alta, de igual forma al enfrentar un problema los participantes poseen la tendencia a expresar sus emociones evitando resolver la dificultada, esto es evidente con el puntaje del factor Emocional-Negativo $(M=5.28)$. Es de singular atención que todos los factores que componen la variable Estilos de Enfrentamiento a la Vida se ubicaron arriba de la media teórica $(M=4)$, que en el continuo del 1 al 7 indica que el adulto mayor utiliza una variedad de estrategias diversas para enfrentar los problemas

Posteriormente para conocer los mejores predictores del Bienestar Subjetivo a partir de la Satisfacción con la Vida, Autopercepción de Salud, el Apoyo Social y los Estilos de Enfrentamiento, se realizó un análisis de regresión múltiple paso a paso por sexo.

En cuanto al análisis de regresión múltiple para hombres no se obtuvo un modelo estadísticamente significativo para el Bienestar Subjetivo en los Afectos Negativos. Por el contrario, como lo muestra la tabla 3 el análisis de regresión múltiple para las mujeres encontró que los Estilos de Enfrentamiento Directo-Revalorativo y Evasivo son buenos predictores del Bienestar Subjetivo en los Afectos Negativos. 


\section{Tabla 2}

Medias, desviaciones estándares y Consistencias internas obtenidas para las Escalas Autopercepción de Salud, Apoyo Social, Satisfacción con la Vida y Estilos de Enfrentamiento

\begin{tabular}{lccc}
\hline Factores & $M$ & $D E$ & $\alpha$ \\
\hline Autopercepción de Salud & 2.96 & .88 & .93 \\
Escala Apoyo social & & & \\
$\quad$ Amistad & 1.87 & .48 & .73 \\
$\quad$ Ausencia de Soledad & 3.03 & .64 & .81 \\
$\quad$ Familia y Esfuerzo & 2.99 & .53 & .49 \\
Escala Satisfacción con la Vida & & & \\
$\quad$ Satisfacción con la Vida & 4.93 & .98 & .85 \\
Escala Estilos de Enfrentamiento & & & \\
$\quad$ Directo-Revalorativo & 5.60 & .70 & .73 \\
$\quad$ Emocional- Negativo & 5.28 & .59 & .29 \\
$\quad$ Evasivo & 5.15 & .69 & .61 \\
$\quad$ Apoyo Social & 5.16 & .73 & .58 \\
\hline
\end{tabular}

\section{Tabla 3}

Análisis de regresión múltiple obtenido para la predicción de la dimensión emocional en sus afectos negativos, a partir de la satisfacción con la vida, autopercepción de salud, apoyo social y estilos de enfrentamiento para mujeres

\begin{tabular}{lcccc}
\hline Factores & $B$ & Beta & $T$ & $p$ \\
\hline Directo-Revalorativo & .292 & .291 & 2.326 & $.02^{*}$ \\
Evasivo & .295 & -.364 & -3.010 & $.004^{* *}$ \\
\hline
\end{tabular}

${ }^{*} p \leq .05 ;{ }^{* *} p \leq .01$

$R=.50, R^{2}=.253$

$F_{(0,536)}=2.147 ; p=.04$ 
El análisis de regresión múltiple para hombres y mujeres en los Afectos Positivos del Bienestar Subjetivo no obtuvo un modelo estadísticamente significativo.

La tabla 4 muestra las regresiones múltiples para en el Bienestar Subjetivo en la dimensión cognitiva para los hombres. Se puede destacar que para este grupo las variables Satisfacción con la Vida, los Estilos de Enfrentamiento Emocional-Negativo, Directo-Revalorativo y Evasivo resultaron ser los mejores predictores para esta dimensión.

\section{Tabla 4}

Análisis de regresión múltiple obtenido para la predicción de la dimensión cognitivo-afectiva, a partir de la satisfacción con la vida, autopercepción de salud, apoyo social y estilos de enfrentamiento para hombres

\begin{tabular}{lcccc}
\hline Factores & $B$ & Beta & $T$ & $p$ \\
\hline Directo-Revalorativo & .441 & .388 & 2.963 & $\mathbf{. 0 0 5}^{* *}$ \\
Emocional-Negativo & .216 & .228 & 2.048 & $\mathbf{. 0 4 6}^{*}$ \\
Evasivo & .191 & .243 & 2.106 & $\mathbf{. 0 4 1}^{*}$ \\
Satisfacción con la Vida & .204 & .342 & 2.550 & $\mathbf{. 0 1 4}^{*}$ \\
\hline${ }^{*} p \leq .05 ;{ }^{* *} \mathrm{p} \leq .01$ & & & & \\
$R=.74, \mathrm{R}^{2}=.55$ & & & & \\
$F_{(9,1143)}=6.191 ; p=.000$ & & & &
\end{tabular}

En cuanto a las mujeres la Autopercepción de Salud, el factor Apoyo Social, la Satisfacción con la Vida y el estilo de enfrentamiento Emocional-Negativo son los mejores predictores del Bienestar Subjetivo en la Dimensión Cognitiva (ver tabla 5). 


\section{Tabla 5}

Análisis de regresión múltiple obtenido para la predicción de la dimensión cognitivo-afectiva, a partir de la satisfacción con la vida, autopercepción de salud, apoyo social y estilos de enfrentamiento para mujeres

\begin{tabular}{lcccc}
\hline Factores & $B$ & Beta & $T$ & $p$ \\
\hline Apoyo Social & .280 & .327 & 3.473 & $.001^{* *}$ \\
Satisfacción con la Vida & .163 & .263 & 2.323 & $.024^{*}$ \\
Autopercepción de Salud & .325 & .453 & 4.375 & $.000^{* *}$ \\
\hline${ }^{*} p \leq .05 ;{ }^{* *} p \leq .01$ & & & & \\
$R=.74, R^{2}=.553$ & & & & \\
$F_{(9,1472)}=7.726 ; p=.000$ & & & &
\end{tabular}

\section{Discusión}

Los resultados encontrados en este estudio en cuanto a satisfacción con la vida y percepción de bienestar subjetivo en los adultos mayores muestran que en general los adultos mayores están satisfechos con su vida y tiene una apreciación positiva del bienestar subjetivo en ambas dimensiones. Estos resultados por una parte coinciden con la literatura (Velasco Matu, 2015), la cual señala que la satisfacción tiende a aumentar o permanecer estable durante la vejez (Kunzman, Little \& Smith, 2000). Diversos estudios han señalado la estabilidad durante el ciclo vital, como una característica principal de la satisfacción (Zika \& Chamberlain, 1992), lo cual no significa que la satisfacción permanezca estable a pesar de las circunstancias cambiantes (Headey \& Wearing, 1989), como señalan Ramírez Pérez y Lee Maturana (2012), quienes comentan que durante el ciclo vital, las variables moderadoras de la satisfacción pueden alterar la forma en que se valora la vida si existen cambios significativos.

Cabe señalar que los estudios sobre el bienestar subjetivo reportan altos niveles en la dimensión cognitiva y menor intensidad en los afectos en los adultos mayores (Diener \& Lucas, 2000). Este estudio 
concuerda con lo señalada en relación a la dimensión cognitiva, sin embargo, difiere en relación a los afectos, al presentar los adultos mayores una apreciación positiva, en donde sobresalen los aspectos emocionales positivos, resaltando el factor afectividad positiva (amor, carińo, felicidad). Estos resultados concuerdan con un estudio realizado en adultos mayores en Yucatán por Peniche Bates (2000) quien encuentra un bienestar subjetivo satisfactorio, ya que los aspectos emocionales positivos predominan sobre los aspectos negativos. En cuanto a la dimensión cognitiva el adulto mayor se encuentra a gusto con la forma en que se relacionan socialmente, con el núcleo familiar con el que conviven y con lo que ha logrado en su vida.

En cuanto a la valoración de la salud, los resultados demuestran que los adultos mayores se perciben con un deficiente estado de salud, situación que se puede explicar por el decaimiento natural de ciertas capacidades funcionales (Aparicio, 2003). Un punto a considerar es que esta valoración negativa de la salud no parece incidir negativamente en el bienestar subjetivo, lo que puede ser explicado por datos de la variable estilos de enfrentamiento. Se observó que la muestra evaluada recurre con mayor frecuencia a la estrategia directa-revalorativa, que consiste en reestructurar la situación de tal forma que se pueda ver el lado positivo y que al final constituya un aprendizaje.

Para explicar lo anterior las teorías del bienestar subjetivo señalan que la capacidad de adaptación es central en la determinación del mismo (Suh, Diener \& Fujita, 1996), es decir esta capacidad para adaptarse actúa como moderadora para compensar las pérdidas percibidas por la edad, manteniendo la valoración de la vida en general como positiva. Estos resultados concuerdan con lo reportado por Vera Noriega, Sotelo Quiñones y Domínguez Guedea (2005), al encontrar que los adultos mayores utilizan con mayor frecuencia un estilo de enfrentamiento a los problemas basado en encuadrar hacia un lado positivo el evento problemático o estresante.

Los resultados relacionados con el apoyo social percibido reportan que los adultos mayores en general perciben un escaso apoyo social (Pi Osoria \& Cobian Mena, 2015), en donde sobresale el factor de 
ausencia de soledad con el mayor puntaje, es decir no se perciben solos, sin embargo, la calidad del apoyo social es insuficiente, al revelar un bajo puntaje los factores de amistad y familia y esfuerzo. Pinazo (2006), subraya la diferencia entre cantidad y calidad de apoyo social y relaciona la palabra calidad con el grado de apoyo afectivo (cariño, solidaridad, amor) e instrumental compañía, cuido, necesidades asistencialistas. Es decir, en este estudio los adultos mayores reportan un menor apoyo social en términos afectivo, lo que concuerda con otros estudios en México, en donde el apoyo social rara vez se relacionó con apoyo emocional o cognitivo (Pelcastre-Villafuerte, Treviño-Siller, González-Vázquez \& Márquez-Serrano, 2011).

En este sentido Castellanos Fuentes (2014) refiere que, a pesar de las bondades del apoyo social, este proceso dista de ser unidireccional, ya que durante el envejecimiento puede sufrir diversas perdidas (Vivaldi \& Barra, 2012) causadas por una gran diversidad de situaciones, entre las que sobresale la viudez, disminución o ausencia del ingreso, pérdida de autonomía, muerte de amistades, cambio de domicilio, institucionalización y jubilación, es así que las fuentes de apoyo social pueden ir mermando paulatinamente, a medida que aumenta la edad

El análisis de regresión múltiple en hombres reveló que las variables satisfacción con la vida, percepción de salud, apoyo social y estilos de enfrentamiento no demuestran capacidad predictiva para la dimensión experiencia emocional del bienestar subjetivo, en cambio para la mujeres las emociones negativas se predicen a través enfrentar de forma activa y tratando de resolver las situaciones estresantes de la vida, así como también expresando emociones y sentimientos que no necesariamente resuelven el problema.

En cuanto a la dimensión cognitiva del bienestar subjetivo, las mujeres obtuvieron como primer predictor a la autopercepción de salud, lo que indica que la valoración global del bienestar está determinada por como concibe su salud, seguidamente por la capacidad de la persona de adaptarse, a través del apoyo que le pueden brindar las personas para solucionar los problemas, asimismo la evaluación de la vida y la expresión de las emociones como una forma de enfrentar situaciones 
estresantes. Para los hombres el bienestar subjetivo en el área cognitiva se predice por la forma en que evalúan su vida y por como enfrentan los problemas, es decir los hombres utilizan mecanismos que le permitan expresar sus emociones, resolver la situación y en ciertas situaciones tratando de evitar el evento estresante.

Un hallazgo a considerar es el papel de la percepción de salud en el bienestar de la mujer mayor, ya que como se observó en los resultados de la regresión múltiple, es la variable con mayor contribución a la predicción del bienestar, lo cual concuerda con los planteamientos que señalan que la salud auto-percibida es un parámetro fundamental en la forma que el adulto mayor valora sus experiencias cognitivas y afectivas de su vida en general, lo que da lugar al bienestar subjetivo (González Celis, 2005; Urzua, Bravo, Ogalde \& Vargas, 2011).

Por su parte en los hombres un aspecto central para el bienestar subjetivo es la capacidad de adaptación, ya que como menciona Carmona Valdés (2011), este proceso provee los recursos necesarios que permitan ser un adulto mayor que participe activamente en la comunidad, adquiriendo nuevas capacidades sociales y así desempeñar su nuevo rol dentro de la sociedad, favoreciendo su autonomía, lo cual guarda cierto sentido en relación con la satisfacción y el bienestar subjetivo, donde las emociones de cada acontecimiento varían en medida en que este se desarrolla y el resultado reflejará las evaluaciones realizadas sobre el menor o el mayor logro de los objetivos del individuo y el grado de satisfacción por sus rendimientos (González-Celis \& Padilla, 2006).

\section{Conclusión}

Los datos demuestran que los adultos mayores, están satisfechos con su vida y tiene altos niveles de bienestar subjetivo lo que concuerda con estudios en otros países (Lucas-Carrasco, 2012; Zubieta, Muratori \& Fernández, 2012; Varela \& Gallegos, 2015). Esto puede ser explicado, desde la óptica de la capacidad adaptativa y también a través de los efectos culturales, como se ha señalado en la literatura Yucatán es un 
ecosistema natural, lo que conlleva a preservar la imagen del adulto mayor (Villagómez Váldez, 2013) como una autoridad de respeto, cuya opinión es matizada por sabiduría, como señala Maas Colli (2013) en un estudio sobre ancianos en comunidades mayas, de tal manera, es necesario continuar y profundizar en el estudio de diversas variables que pueden impactar en el bienestar de los adultos mayores.

Algunas de las consideraciones y recomendaciones de este estudio sería el considerar el número de reactivos administrados en las diferentes escalas a los participantes, dado que este fue muy extenso. En este sentido los participantes refirieron dificultades para contestarlo debido al cansancio, de ahí que en algunos casos fue necesario administrarlos en forma personal, por lo que se sugiere reducir aún más los instrumentos de medición o bien en el futuro realizar dado las características de la muestra y la dificultad de manera independiente poder contestar un instrumento de lápiz y papel por sus condiciones de salud minimizadas (psicomotoras, visuales y auditivas) se pueda realizar un estudio mediante técnicas cualitativas que permitan con mayor funcionalidad en los adultos mayores poder compartir sus creencias, emociones y conductas en la etapa que están actualmente viviendo.

También es relevante para el estudio señalar algunas limitaciones a considerar en el futuro, tales como el tamaño de la muestra que fue limitada, debido a las dificultades para ubicar a los participantes, así como también por su estado de salud mental o físico, lo cual no permitió que participaran más personas en el estudio.

Finalmente, es fundamental considerar al bienestar subjetivo multifactorial, lo que invita caracterizar las variables que covarían con los distintos aspectos del bienestar subjetivo en diversos contextos culturales, y en personas de todos los sectores sociales (Celemin, Mikkelsen \& Velázquez, 2015). De igual forma, un aporte valioso sería conocer los significados que atribuyen los adultos mayores al bienestar subjetivo.

Uno de los ideales de la investigación es crear un conocimiento del bienestar subjetivo, cuya validez cultural promueva un envejecimiento saludable, que finalmente se extienda hacia el envejecimiento exitoso, para los adultos mayores de Yucatán y todo México y en el mundo. 


\section{Referencias}

Acevedo Alemán, J., González Tovar, J. (2014). No envejecemos igual: la religiosidad y el género en adultos mayores del noreste de México. Reflexiones, 93, 133-144. Recuperado de http://www. redalyc.org/articulo.oa?id=72930086010

Alvarado García, A. M. y Salazar Maya, A. M. (2014). Análisis del concepto del envejecimiento. Gerokomos, 25(2), 57-62. https:// doi.org/10.4321/S1134-928X2014000200002

Anguas Plata, A. M. (1997). El significado del bienestar subjetivo, su valoración en México. (Tesis de maestría). México, D.F.: Universidad Nacional Autónoma de México.

Anguas Plata, A. M. (2000). El bienestar subjetivo en la cultura mexicana. (Tesis de doctorado). Universidad Nacional Autónoma de México. México, D.F.

Anguas Plata, A. M. \& Reyes Lagunes, I. (1999). Conformción estructual del bienestar subjetivo. El caso específico de los yucatecos. Revista Sonorense de Psicología, 13(22), 15-22. Recuperado de http://kunkaak.psicom.uson.mx/rsp/13-2-15.pdf

Aparicio, R. (2003). Transición demográfica y vulnerabilidad durante la vejez. En CONAPO, La situación demográfica de México (pp. 155-168). México, D.F.

Aranda, J. \& Vara, A. (2006). Factores asociados a la satisfacción de vida de los adultos mayores de 60 años en Lima-Perú. Universitas Psychologica, 5(3), 37-53. Recuperado de http://www.scielo. org.co/pdf/rups/v5n3/v5n3a04.pdf

Aranibar, P. (2004). Calidad de vida y vejez. Universidad de Barcelona. Recuperado de http://webcache. googleusercontent.com/search?q=cache:IoSzLDVAH-cJ:www. ciape.org.br/artigos/calidaddevidayvejez\%255B1\%255D. $\mathrm{doc}+\& \mathrm{~cd}=2 \& \mathrm{hl}=\mathrm{es} \& \mathrm{ct}=\mathrm{clnk} \& \mathrm{gl}=\mathrm{mx}$

Aranibar, P. (2001). Acercamiento conceptual a la situación del adulto mayor en América Latina. Chile: CEPAL. 
Arias, C. (2008).Elapoyosocialenlavejez:alternativasdeacciónfrentealos desafíos del envejecimiento poblacional. Perspectivas en Psicología, 5(2), 54-61. Recuperado de https://psicsocialcomunitaria.files. wordpress.com/2014/05/el-apoyo-social-en-la-vejez-alternativasde-accic3b3n-frente-a-los-desafc3ados-del-envejecimientopoblacional.pdf

Arroyo Rueda, M. C. (2011). Sentirse una carga en la vejez. Kairos Gerontologia, 6(14), 5-29. Recuperado de http://seminarioenvejecimiento.unam.mx/Publicaciones/articulos/sentirse_carga.pdf

Caicedo, B.\& y Berbesi Fernández, D. (2014). Salud autorreferida: influencia de la pobreza y la desigualdad del área de residencia. Gaceta Sanitaria, 29(2), 97-104. https://doi.org/10.1016/j. gaceta.2014.11.007

Calvo Francés, F. \& Díaz Palarea, M. (2004). Apoyo social percibido: Características psicométricas del cuestionario Caspe en una población urbana geriátrica. Psicothema, 16(4), 570-575.

Cano, F., Rodríguez, L. \& García, J. (2007). Adaptación española del Inventario de Estrategias de Afrontamiento. Revista Actas Españolas de Psiquiatría, 35, 29-39.

Carrasco-Campos, A., Martínez Fernández, L. C. \& Moreno Mínguez, A. (2013). Revisión crítica de la medición del bienestar desde una perspectiva interdisciplinar: Hacia una propuesta de indicadores subjetivos y espaciales. Prisma Social, 91-122. Recuperado de http://www.redalyc.org/articulo.oa?id=353744535004

Carmona Valdez, S. (2011). El bienestar personal en el envejecimiento. Revista de Ciencias Sociales de la Universidad Iberoamericana, 7 , 48-65.

Castellano Fuentes, C. L. (2014). La influencia del apoyo social en el estado emocional y las actitudes hacia la vejez y el envejecimiento en una muestra de ancianos. International Journal of Psychology \& Psychological Therapy, 14(3), 365-377.

Castro Bolaño, C., Otero-López, J. M., Freire Garabal, M., Núñez Iglesias, M. J., Losada Vázquez, C., Saburido Novoa, J. L. \& Pereiro Raposo, D. (1995). Un estudio comparativo de las estrategias 
de afrontamiento al estrés en distintos grupos de edad. Revista Española de Geriatría y Gerontología, 30(2), 73-78.

Celemin, J., Mikkelsen, C. \& Velázquez, G. (2015). La calidad de vida desde una perspectiva geográfica: integración de indicadores objetivos y subjetivos. Revista Universitaria de Geografia, 24(1), 63-84. Recuperado de http://www.scielo.org.ar/scielo. php?script=sci_arttext\&pid=S1852-42652015000100004\&lng $=$ es\&tlng=en.

Cerquera Córdoba, A., Flores Jaimes, L. \& Linares Restrepo, M. (2010). Autopercepcióndela salud en el adulto mayor. Revista Virtual UniversidadCatólicadelNorte,31(11),407-428. Recuperadodehttp:// revistavirtual.ucn.edu.co/index.php/RevistaUCN/article/view/ $51 / 111$

Chacón, F. (2015). Índices de Felicidad y Bienestar. Serie Indicadores Internacionales de Desarrollo, 6, 1-27.

Consejo Nacional para la Prevención de la Discriminación. (2010). Encuesta nacional sobre la discriminación en México. México: INEDIM.

Csikszentmihalyi, M. (1998). Creatividad el fluir y la psicología del descubrimiento y la invención. Barcelona: Paidós.

Davis, S. y Palladino, J. (2008). Psicología. México: Pearson Prentice Hall.

Deluigi, R. (2016). Ageing, transnational families, and elderly care strategies: social interactions, welfare challenges and equitable well-being. Rivista Italiana di Educazione Fami, 2, 19-32. https://doi.org/10.13128/RIEF-19518

Diener, E. \& Chan, M. (2011). Happy People Live Longer: Subjective Well-Being Contributes to Health and Longevity. Applied Psychology: Health Benefits of Happiness, 3(1), 1-43. https://doi. org/10.1111/j.1758-0854.2010.01045.x

Diener, E., Emmons, R. A., Larson, R. J. \& Grifffin, S. (1985). The satisfaction with life scale. Journal of Personality Assesment, 49(1), 71-75. https://doi.org/10.1207/s15327752jpa4901_13 
Diener,E., Kesebir,P. \&Lucas, R.(2008). Benefitsofaccountsofwell-being For Societies and for Psychological Science. Applied Psychology, 57, 37-53. https://doi.org/10.1111/j.1464-0597.2008.00353.x Diener, E. \& Lucas, R. (1999). Personality, and subjective well-being. En D. Kahneman, E. Diener y N. Schwarz, N. (Eds.), Wellbeing: The foundations of hedonic psychology (pp. 213-229). New York: Russell Sage Foundation

Diener, E. \& Suh, E. (1998). Age and subjetive well-being: an international analysis. Annual Review of Gerontology and Geriatrics, 15, 304-324.

Fernández-Ballesteros, R. (1996). Psicología del envejecimiento: crecimiento y declive. Madrid: Universidad Autónoma de Madrid.

Fernández-Ballesteros, R., Moya Fresneda, R., Ińiguez Martínez, J. y Zamarrón, M.D. (1999). Qué es la psicología de la vejez. Madrid: Biblioteca Nueva.

Figueroa, M. I., Contini, N., Lacunza, A., Levín, M. \& Estévez, A. (2005). Las estrategias de afrontamiento y su relación con el nivel de bienestar psicológico. Un estudio con adolescentes de nivel socioeconómico bajo de Tucumán, Argentina. Anales de Psicología, 21(1), 66-72.

Florenzano, R. y Dussaillant, F. (2012). Felicidad, salud física y salud mental: evidencia empírica en Chile. En R. Alarcón (Ed.), Psicología positiva. La Molina, Perú: Universidad Femenina del Sagrado Corazón.

Folkman, S., Lazarus, R., Pimley, S. \& Novacek, J. (1987). Age differences in stress and coping processes. Psychology and Aging, 2(2), 171-184. https://doi.org/10.1037/0882-7974.2.2.171

Folkman, S. \& Moskowitz, J. T. (2004). Coping: Pitfalls and promise. Annual Review of Psychology, 55, 745-774. https://doi. org/10.1146/annurev.psych.55.090902.141456

Gallegos-Carrillo, K., García-Peña, C., Reyes, H. \& Duran, L. (2006). Autopercepción del estado de salud: Una aproximación a los ancianos en México. Revista de Salud Pública, 40, 792-801. https://doi.org/10.1590/S0034-89102006000600008 
Garay Villegas, S. \& Montes de Oca Zavala, V. Z. (2012). La vejez en México: una mirada general sobre la situación socioeconómica y familiar de los hombres y mujeres adultos mayores. Perfiles Sociales, 13(1), 1-22.

García Martín, M. (2002). Desde el concepto de felicidad al abordaje de las variables implicadas en el bienestar subjetivo: un análisis conceptual. Revista Digital Buenos Aires, 48, 1-6.

García Vigara, C. (2015). El bienestar subjetivo y su relación con la salud. Un análisis para España. (Tesis de maestría). Universidad de Valladolid. Valladolid, España.

Góngora Coronado, E. \& Reyes Lagunes, I. (1999). La estructura de los estilos de enfrentamiento: Rasgo y Estado en un ecosistema tradicional mexicano. Revista Sonorense de Psicología, 13(2), 3-14. Góngora Coronado, E. A. \& Reyes Lagunes, I. (2000). El enfrentamiento a los problemas y locus de control. La Psicología Social en México, 3, 165-172.

González-Celis, R. (2005). ¿Cómo mejorar la calidad de vida y el bienestar subjetivo de los ancianos? En L. Garduño, B. Salinas y M. Rojas (Coords.). Calidad de Vida y Bienestar Subjetivo en México. (pp. 259-294). México: UDLA Puebla-Plaza y Valdés.

Gonzalez-Celis, R. A. \& Araujo, V. A. (2010). Estrategias de afrontamiento ante la muerte y calidad de vida en adultos mayores. Revista Kairós Gerontologia, 13(1), 167-90. Recuperado de http:// revistas.pucsp.br/index.php/kairos/article/viewFile/4868/3450

González-Celis, A. L. \& Padilla, A. (2006). Quality of life and coping strategies in older adults with problems and diseases in Mexico city. Universitas Psychologica, 5(3), 501-509. Recuperado de http://www.scielo.org.co/scielo.php?script=sci_arttext\&p id $=$ S1657-92672006000300006

Gonzales de Gago, J. (2010). Teorías del envejecimiento. Tribuna del Investigador, 1(11), 42-66. Recuperado de http://datateca.unad. edu.co/contenidos/301138/Teorias_del_envejecimiento.pdf

Gwozdz, W. \& Sousa-Poza A. (2010). Ageing, Health and Life Satisfaction of the Oldest Old: An Analysis for Germany. 
Discussion Papers Series, 4053, 1-37. https://doi.org/10.1007/ s11205-009-9508-8

Ham Chande, R. (2011). Diagnóstico sociodemográfico del envejecimiento en México. En La situación demográfica de México 2011, Consejo Nacional de Población. Recuperado de http://www. conapo.gob.mx/es/CONAPO/Diagnostico_socio_demografico_del_envejecimiento_en_Mexico >

Headey, B.W. \& Wearing, A.J. (1989). Personality, life events and subjective well-being: Toward a dynamic equilibrium model, Journal of Personality and Social Psychology, 57, 731-39. https:// doi.org/10.1037/0022-3514.57.4.731

Hebrero Martinez, M. (2010). Salud y bienestar en la población adulta mayor. En B. Montoya Arce \& H. Montes de Oca Vargas (Eds.), Análisis sociodemográfi co del envejecimiento en el Estado de México (pp. 109-145). Toluca: UAEM.

Herzog, A. W., Rogers J. \& Woodworth, J. (1983). Subjective wellbeing among different age groups. Ann Arbor, MI: Survey Research Center, Institute for Social Research, The University of Michigan.

Hofer, S. \& Sliwinski, M. (2006). Design and analysis of longitudinal studies on. En J. Birrin \& K. Schaie (Eds.), Handbook of the psychology of aging (pp. 15-37). San Diego: Elsevier Academic Press. https://doi.org/10.1016/B978-012101264-9/50005-7

Instituto Nacional de Estadística, Geografía e Información (2013). Conteo de población y vivienda.

Instituto Nacional de Estadística, Geografía e Información (2013). Estadísticas a propósito del día Internacional de las personas de edad datos de Yucatán. Mérida: INEGI.

Kehl, S. \& Férnandez, M. (2001). La construcción social de la vejez. Cuadernos de Trabajo Social,14, 125-161.

Krause, N. (1997). Anticipated support, received support, and economic stress among. Journal of Gerontology: Psychological Sciences, 52, 284-293. https://doi.org/10.1093/geronb/52B.6.P284 
Krause, N., Liang, J. \& Yatomi, N. (1989). Satisfaction with social support and depressive symptoms: A panel analysis. Psychology and Aging, 4, 88-97. https://doi.org/10.1037/0882-7974.4.1.88

Kunzmann, U., Little, T. \& Smith, J. (2000).Is age-related stabilidad of subjetive well-being a paradox? Cross-sectional and logitudinal evidence from evidence from Berlin Aging Study, Psychology and Aging, 15(3), 511-526. https://doi. org/10.1037/0882-7974.15.3.511

Laforest, J. (1991). Introducción a la Gerontología. Barcelona: Herder.

Lazarus, R.S. \& Folkman, S. (1984). Estrés y procesos cognitivos. Barcelona: Martínez Roca.

Lepore, S. J., Evans, G. W. \& Schneider, M. (1991). Dynamic role of social support in the link between chronic stress and psychological distress. Journal of Personality and Social Psychology, 61, 899-909. https://doi.org/10.1037/0022-3514.61.6.899

Lin, N. \& Ensel, W. (1989). Life stress and health: Stressors and resources. American Sociological review, 54, 382-389. https://doi. org/10.2307/2095612

Lucas-Carrasco, R. (2012). Reliability and validity of the Spanish version of the world health organization-five well-being index in elderly. Psychiatry and Clinical Neurosciences, 66, 508-513. https://doi.org/10.1111/j.1440-1819.2012.02387.x

Máas Collí, H. (2013). Costumbres de respeto y trato a los nukuch máako'ob. En G. Villagomez Valdez y L. Vera Gamboa (Coords.), Vejez una perspectiva sociocultural (pp. 47-62). México: UADY, UAC

Mella, R., Apolonio, J., Maldonado, I., Fuenzalida, A. \& Díaz, A. (2004). Factores asociados al bienestar subjetivo en el adulto mayor. Pshyque, 13, 79-89. https://doi.org/10.4067/ S0718-22282004000100007

Mendoza Sierra, M., Revilla Delgado, C., Feria Muñoz, A., Bernal Vilán, J., Barbero Prado, M. \& Revilla Parody, T. (2004). La calidad de vida de las personas mayores en una zona de salud 
de Huelva. Portularia, 4, 199-208. Recuperado de http://rabida. uhu.es/dspace/bitstream/handle/10272/174/b15134258.pdf?s

Montes de Oca Zavala, V. (2003). El envejecimiento en el debate mundial: reflexión académica y política. Papeles de Población, 35, 77-100.

Montes de Oca, V., (2013) La discriminación hacia la vejez en la ciudad de México: contrastes sociopolíticos y jurídicos a nivel nacional y local. Perspectivas Sociales, 15(1), 47-80. Recuperado de http:// seminarioenvejecimiento.unam.mx/Publicaciones/articulos/ discriminacion_vejez_cd.pdf

Muñoz Muñoz, C. (2013). Bienestar subjetivo y actividad social con sentido histórico en adultos mayores. Hacia la Promoción de la Salud, 18(2), 13-26. Recuperado de http://www.redalyc.org/ pdf/3091/309131077002.pdf

Newsom, J. T., Mahan, T. L., Rook, K. S. \& Krause, N. (2008). Stable negative social exchanges and health. Health Psychology, 27, 78-86. https://doi.org/10.1037/0278-6133.27.1.78

Olvera, M. (Noviembre de 2015). El bienestar subjetivo y su relación con personalidad y autoestima. En A. Viguera (Presidencia), V Congreso Internacional de Investigación de la Facultad de Psicología de la Universidad Nacional de La Plata. Congreso llevado a cabo en La Plata, Argentina.

Organización Mundial de la Salud (2015). Informe Mundia sobre el Envejecimiento y la Salud. Estado Unidos de America: OMS.

Orthner, D. K. \& Mancini, J. A. (1990). Leisure impacts on family interaction and cohesion. Journal of Leisure Research, 22(2), $125-137$.

Pelcastre-Villafuerte, B., Treviño-Siller, S., González-Vázquez, T. \& Márquez-Serrano, M. (2011). Social support and living conditions in poor elderly people in urban Mexico. Cad Saúde Pública. 27(3), 460-70. https://doi.org/10.1590/ S0102-311X2011000300007

Pelechano, V. (2000). Psicología sistemática de la personalidad. Barcelona: Ariel. 
Pi Osoria, A. \& Cobián Mena, A. (2015). Desarrollo del clima familiar afectivo y su impacto en el bienestar subjetivo de la familia. Revista Médica Granma, 19(2), 1-13.

Pinazo, S. (2006). El apoyo social y las relaciones sociales de las personas mayores en gerontologia: actualización, innovación y propuestas. Madrid: Pearson Prentice Hall.

Pinquart, M,. \& Sörensen, S. (2000). Influences of socieconomic status, social network and competence on subjetive well-beging in later life: a meta-analysis. Psychology and Aging, 15(2), 187224. https://doi.org/10.1037/0882-7974.15.2.187

Quintero, Á., Henao, M., Villamil, M. \& León, J. (2015). Cambios en la depresión y el sentimiento de soledad después de la terapia de la risa en adultos mayores internados. Biomédica, 35(1), 90-100. https://dx.doi.org/10.7705/biomedica.v35i1.2316

Ramírez Pérez, M. \& Lee Maturana, S. (2012). Factores asociados a la satisfacción vital en adultos mayores de 60 años. Revista de la Universidad Bolivariana, 11(33), 407-427. Recuperado de http://www.scielo.cl/pdf/polis/v11n33/art20.pdf

Ramiro, P., Navarro, J., Menacho Jiménez, I., Mar López, M. \& Garcia-Sedeño, M. (2016). Bienestar psicológico en personas con alta capacidad intelectual/Psychological well-being in gifted people. European Journal of Education and Psychology, 9, 72-78. https://doi.org/10.1016/j.ejeps.2015.12.001

Razo González, A. (2014). La política pública de vejez en México: de la asistencia pública al enfoque de derechos. Revista Conamed, 19(2), 78-85. Recuperado de https://dialnet.unirioja.es/descarga/articulo/4760916.pdf

Reyes Rasse, C. \& Rojas Arellano, P. (2006). Las estrategias de afrontamiento frente a la percepción de apoyo social: Estudio Descriptivo-Comparativo con adultos mayores institucionalizados y no institucionalizados de la Región Metropolitana, considerando la variable género. SUMMA Psicológica, 1(1), 77-82. 
Roig Hechavarría, C., Avila Fernandez, E., Mcdonald Bron, D. \& Avila Fernandez, B. (2015). La atención al adulto mayor: un reto para la medicina contemporánea. Revista Cubana de Medicina General Integral, 31(3), 346-352. Recuperado de http://www. medigraphic.com/pdfs/revcubmedgenint/cmi-2015/cmi153i.pdf Rojpaisarnkit, K. (2016). Factors influencing well-being in the elderly living in the rural areas of eastern thailand. International Journal of Behavioral Science, 12(2), 31-50.

Romero Ruvalcaba, J. (2005). Discriminación y adultos mayores: un reto mayor. El Cotidiano, 134, 56-63.

Salas, C.,\& Garzón, M. (2013). La noción de calidad de vida y su medición. Salud Pública, 4, 36-46. Recuperado de https:// search.proquest.com/openview/ae85ec5dc7818ff0d69a94827 $-+\mathrm{a} 0 \mathrm{bd} 1 \mathrm{ab} / 1$ ?pq-origsite $=$ gscholar $\& \mathrm{cbl}=2037661$

Sandin B (2003). El estrés: un análisis basado en el papel de los factores sociales. International Journal of Clinical and Health Psychology, 3, 141-157. Recuperado de http://www.aepc.es/ ijchp/articulos_pdf/ijchp-65.pdf

Salinas, A., Manrique, B. \& Téllez-Rojo, M. (24 de septiembre de 2008). Redes de apoyo social: adultos mayores beneficiarios del componente de adultos mayores del programa oportunidades. 161-179.

Salinero Fort, M.A. (2013). Autopercepción de la salud, apoyo social y salud mental en Atención Primaria: diferencias entre población autóctona e inmigrante. (Tesis de doctorado). Universidad Rey Juan Carlos. Madrid, España.

Seligman, M. (2013). Lourish, a visionary new understanding of happiness and well-being. New York: Free Press/Simon and Schuster.

Solomon, R. (1996). Coping with stress: A physician's guide to mental health in aging. Geriatrics, 7(51), 46-51.

Stefani, D. (2004). Gender and coping style in old age. Revista de Psicología y Ciencias Afines, 99, 109-125. Recuperado de http:// www.redalyc.org/articulo.oa?id=18009908 
Stefani, D. \& Feldberg, C. (2006). Estrés y estilos de afrontamiento en la vejez: Un estudio comparativo en senescentes argentinos institucionalizados y no institucionalizados. Anales de Psicologia, 22(2), 267-272. Recuperado de http://revistas.um.es/ analesps/article/viewFile/25951/25191

Stein, A.C. (2016). Relación entre actividad fisica, salud percibida, bienestar subjetivo, depresión y enfermedades crónicas en personas mayores. (Tesis de doctorado). Universidad de León. León, México.

Steptoe, A, Deaton, A. \& Stone, A. (2015). Subjective wellbeing, health, and ageing. Lancet, 385, 640-648. https://doi.org/10.1016/ S0140-6736(13)61489-0

Suarez, R. \& Pescetto, C. (2005). Sistemas de protección social para el adulto mayor en América Latina y el Caribe. Revista Panam Salud Publica, 5(17), 419-428. https://doi.org/10.1590/ S1020-49892005000500014

Suh, E., Diener, E. \& Fujita, F. (1996). Events and subjective well-being. Only recentes events matter. Journal of Personality and Social Psychology, 70, 1091-1102. https://doi. org/10.1037/0022-3514.70.5.1091

Svensson, A, Martensson, L. \& Hellstrom Muhli, U. (2012). Wellbeing dialogue: elderly women's subjective sense of well-being from their course of life perspective. International Journal Qualitative Stud Health Well-being, 7(1), 192-207. https://doi. org/10.3402/qhw.v7i0.19207

Temkin, B. \& Del Tronco, J. (2006). Desarrollo humano, bienestar subjetivo y democracia: confirmaciones, sorpresas e interrogantes. Revista Mexicana de Sociologia, 68(4), 731-760.

Torres Gongóra, B. (2013). Antes y después del cese laboral. Experiencias y expectativas de trabajadores mayores en la ciudad de Mérida. En G. Villagómez Váldez \& L. Vera Gamboa (Eds.), Vejez. Una perspectiva sociocultural (pp. 151-179). México: Universidad Autónoma de Yucatán-Universidad Autónoma de Campeche. 
Triadó, C. (2003). Envejecer en entornos rurales. Madrid: Imserso. Recuperado de http://envejecimiento.csic.es/documentos/ documentos/imserso-estudiosidi-19.pdf

Tu, Y. \& Yang, Z. (2015). Self-Control as mediator and moderator of the relationship between social support and subjective wellbeing among the chinese elderly. Social Indicators Research, 126, 813-828. https://doi.org/10.1007/s11205-015-0911-z

Urzúa, A., Bravo, M., Ogalde, M. \& Vargas, C. (2011). Factores vinculados a la calidad de vida en el adulto mayor. Rev Med Chile, 139, 1006-1014. https://doi.org/10.4067/S003498872011000800005

Varela, L. L. \& Gallego, A. E. (2015). Percepción de la calidad de vida en un grupo de adultos mayores de Envigado (Colombia). Salud \& Sociedad, 2(1). Recuperado de http://revistas.uptc. edu.co/revistas/index.php/salud_sociedad/article/view/3974

Vargas Esquivel, L. (2002). Autopercepción de la salud, funcionalidad familiar y social de una población suburbana de adultos mayores. Revista Especializada en Ciencias de la Salud, 5(1), 20-32. Recuperado de http://www.revistas.unam.mx/index. $\mathrm{php} / \mathrm{vertientes/article/view/33232/30416}$

Veenhoven, R. (2012). World Database of Happiness. Erasmus University Rotterdam, Netherlands. Recuperado de http:// worlddatabaseofhappiness.eur.nl

Velasco Matu, P. (2015). Una aproximación bio-psico-socio-cultural al estudio del Bienestar Subjetivo en México: un estudio explicativo (Tesis doctoral). Universidad Autónoma de México, D.F. México.

Vera, J. \& Sotelo, T. (2004). Bienestar y redes de apoyo social en personas de la tercera edad. La Psicología Social en México, (10), 95-102.

Vera Noriega, J., Quiñones Sotelo, T. \& Domínguez Guedea, M. (2005). Bienestar subjetivo, enfrentamiento y redes de apoyo social en adultos mayores. Revista Intercontinental de Psicología 
y Educación, 7, 57-78. Recuperado de http://www.redalyc.org/ $\mathrm{pdf} / 802 / 80270205 . \mathrm{pdf}$

Villagómez Váldez, G. (21 de octubre de 2013). Vejez en la pobreza. La situación del adulto mayor sin cambio en Yucatán. Diario de Yucatán, pp. 12-13.

Villagómez Váldez, G. \& Sánchez González, M. (2014). Mujeres mayas, envejecimiento y pobreza. Peninsula, 9(2), 75-98. https://doi. org/10.1016/S1870-5766(14)71801-X

Vivaldi, F. \& Barra, E. (2012). Bienestar psicológico, apoyo social percibido y percepción de salud en adultos mayores. Terapia Psicológica, 30(2), 23-29. https://doi.org/10.4067/ S0718-48082012000200002

Zika, S. \& Chamberlain, K. (1992). On the relationbetween meaning in life and psychological well-being. British Journal of Psychology, 83, 133-145. https://doi.org/10.1111/j.2044-8295.1992.tb02429.x

Zubieta, M., Muratori, M. \& Fernandez, O. (2012). Bienestar subjetivo y psicosocial: explorando diferencias de género. Salud y Sociedad, 3(1), 66-76. https://doi.org/10.22199/ S07187475.2012.0001.00005

Zúńiga Herrera, E. \& García, J. E. (2008). El envejecimiento demográfico en México. Principales tendencias y características. Horizontes, 13 , 93-100.

Wong, R. (2013). México envejece. Informador. Recuperado de http:// www.informador.com.mx/suplementos/2013/488434/6/ mexico-envejece-prematuramente-segun-el-inegi.htm

Recibido: 23 de junio, 2016

Revisado: 2 de marzo, 2017 Aceptado: 27 de septiembre, 2017 